\title{
Promoting effect of small molecules in cardiomyogenic and neurogenic differentiation of rat bone marrow- derived mesenchymal stem cells
}

This article was published in the following Dove Press journal:

Drug Design, Development and Therapy

24 December 2015

Number of times this article has been viewed

\author{
Ramin Khanabdali' \\ Anbarieh Saadat' \\ Maizatul Fazilah' \\ Khairul Fidaa' Khairul Bazli' \\ Rida-e-Maria Qazi ${ }^{2}$ \\ Ramla Sana Khalid ${ }^{2}$ \\ Durriyyah Sharifah Hasan \\ Adli' \\ Soheil Zorofchian \\ Moghadamtousi' \\ Nadia Naeem ${ }^{2}$ \\ Irfan Khan ${ }^{2}$ \\ Asmat Salim² \\ ShamsulAzlin Ahmad \\ Shamsuddin' \\ Gokula Mohan' \\ 'Institute of Biological Sciences, \\ Faculty of Science, University of \\ Malaya, Kuala Lumpur, Malaysia; \\ ${ }^{2}$ Dr Panjwani Center for Molecular \\ Medicine and Drug Research, \\ International Center for Chemical \\ and Biological Sciences, University \\ of Karachi, Karachi, Pakistan
}

Correspondence: Gokula Mohan Institute of Biological Sciences, Faculty of Science, University of Malaya, 50603 Kuala Lumpur, Malaysia

Tel +60 379674363

Fax +60379674178

Email g.mohan@um.edu.my

Asmat Salim

Dr Panjwani Center for Molecular Medicine and Drug Research,

International Center for Chemical and Biological Sciences, University of Karachi, Karachi 75270, Pakistan

Email asmat.salim@iccs.edu

\begin{abstract}
Small molecules, growth factors, and cytokines have been used to induce differentiation of stem cells into different lineages. Similarly, demethylating agents can trigger differentiation in adult stem cells. Here, we investigated the in vitro differentiation of rat bone marrow mesenchymal stem cells (MSCs) into cardiomyocytes by a demethylating agent, zebularine, as well as neuronal-like cells by $\beta$-mercaptoethanol in a growth factor or cytokines-free media. Isolated bone marrow-derived MSCs cultured in Dulbecco's Modified Eagle's Medium exhibited a fibroblast-like morphology. These cells expressed positive markers for CD29, CD44, and CD117 and were negative for CD34 and CD45. After treatment with $1 \mu \mathrm{M}$ zebularine for 24 hours, the MSCs formed myotube-like structures after 10 days in culture. Expression of cardiac-specific genes showed that treated MSCs expressed significantly higher levels of cardiac troponin-T, Nkx2.5, and GATA-4 compared with untreated cells. Immunocytochemical analysis showed that differentiated cells also expressed cardiac proteins, GATA-4, Nkx 2.5, and cardiac troponin-T. For neuronal differentiation, MSCs were treated with 1 and $10 \mathrm{mM} \beta$-mercaptoethanol overnight for 3 hours in complete and serum-free Dulbecco's Modified Eagle's Medium, respectively. Following overnight treatment, neuron-like cells with axonal and dendritic-like projections originating from the cell body toward the neighboring cells were observed in the culture. The mRNA expression of neuronal-specific markers, Map2, Nefl, Tau, and Nestin, was significantly higher, indicating that the treated cells differentiated into neuronal-like cells. Immunostaining showed that differentiated cells were positive for the neuronal markers Flk, Nef, Nestin, and $\beta$-tubulin.
\end{abstract}

Keywords: MSCs, cardiomyocytes, demethylating agent, zebularine, neuron-like cells, $\beta$-mercaptoethanol

\section{Introduction}

Heart and neurological diseases are among the leading cause of morbidity and mortality worldwide. The main condition is the inability of the infarcted heart and brain tissue for self-renewal. ${ }^{1-5}$ Diseases such as myocardial infarction and Parkinson's disease are characterized by irreversible loss of specific cell types, thus leading to tissue and organ dysfunction. Stem cell transplantation is one of the most applicable treatment modalities proposed to improve the outcome of patients with heart failure and neuronal diseases. The multilineage differentiated potential of stem cells has opened new horizons in the field of regenerative medicine. In recent years, an increasing body of research suggests that adult stem cells, particularly bone marrow-derived mesenchymal stem cells (BM-MSCs), are multipotent and are capable of transdifferentiating across tissue lineage boundaries into mature cell types other than their tissue of origin. ${ }^{6,7}$

BM-MSCs have been shown to ameliorate tissue damage and improve heart function after myocardial infarction, ${ }^{8,9}$ lung injury, ${ }^{10,11}$ kidney disease,,${ }^{12,13}$ diabetes, ${ }^{14,15}$ liver

submit your manuscript $\mid$ www.dovepress.con 
injury, ${ }^{16,17}$ and neurological disorder. ${ }^{18,19}$ Several studies have shown that MSCs are a promising therapeutic option for the treatment of heart and neurological disorders. ${ }^{20-26}$ However, inappropriate supply of cells during cell therapy to the target organ is of main concern, and it is difficult to deliver the cells to the site of injury. One alternative strategy is the generation of predifferentiated cells into appropriate cell types in vitro and then transplant them in vivo.

Many agents such as cytokines, growth factors, neurotrophins, and small molecules have been shown to promote neuronal and cardiac cell differentiation both in vivo and in vitro. ${ }^{27-29}$ Woodbury et $\mathrm{al}^{4}$ showed the positive effect of some antioxidants, dimethyl sulfoxide, butylated hydroxyanisole, and $\beta$-mercaptoethanol (BME), on the differentiation of BM-MSCs into neuronal cells. Among all the inducing agents, BME and 5-azacytidine appeared to be well-known agents for stem cell differentiation into neuronal cells and cardiomyocytes, respectively. BME is considered as one of the significant inducers since it could not only induce neuronal differentiation morphologically but also induce the expression of neuronal markers. ${ }^{4}$ Several studies reported that BM-MSCs can be induced with 5-azacytidine to express cardiac-specific markers and exhibit spontaneous beating and measurable action potential, consistent with a myocyte lineage..$^{22,28,30-33}$ However, 5-azacytidine is toxic in vitro and in vivo and has been difficult to administer due to its low stability in aqueous solution. Zebularine is also another DNA methyltransferase inhibitor, which is more stable and less toxic. ${ }^{34}$ Although there are several reports of using 5-azacytidine, there is little evidence of using zebularine as an inducer of cardiac differentiation. More studies need to be done in order to investigate the potential of this compound for stem cells differentiation. In this study, the cardiomyogenic and neuronal differentiation potential of BM-MSCs was investigated in response to zebularine and BME treatment, respectively, without using any growth factors or cytokines. Our main objective was to use a single compound for differentiation, whereas other studies had used coculture or multiple compounds. Various concentrations of the compound were tested for different time periods in culture. This study will provide a simple and convenient strategy for the differentiation of MSCs into cardiac- and neuronal-like cells.

\section{Materials and methods}

\section{Isolation and culturing of BM-MSCs}

Bone marrows for isolation of MSCs were obtained from adult Sprague Dawley rats weighing 200 to 250 g. Procedures for sacrificing the animals were performed in accordance with the guidelines for animal experimentation by Institutional Animal Care and Use Committee and were approved by the local ethical committee. Tibia and femur bones were dissected out and subsequently cleaned of muscle and connective tissues. Bone marrow aspirate was cultured in complete Dulbecco's Modified Eagle's Medium (DMEM) (Thermo Fisher Scientific, Waltham, MA, USA), supplemented with $10 \%$ (v/v) fetal bovine serum (Biowest, Nuaillé, France), $100 \mathrm{U} / \mathrm{mL}$ penicillin, and $0.1 \mathrm{mg} / \mathrm{mL}$ streptomycin (Thermo Fisher Scientific), and incubated in a humidified atmosphere with $5 \% \mathrm{CO}_{2}$ at $37^{\circ} \mathrm{C}$. Nonadherent hematopoietic cells were removed using frequent medium change. During the expansion and maintenance of BMMSCs, the medium was replaced three times per week, and the cells were subcultured after they reached $70 \%$ confluence.

\section{Characterization of MSCs}

\section{Immunocytochemistry}

Passage 2 MSCs were grown on chambered slides overnight, washed with phosphate-buffered saline (PBS), and fixed for 20 minutes with $4 \%$ paraformaldehyde at room temperature. Cells were then rinsed with PBS three times for 3-5 minutes. The fixed cells were then permeabilized with $0.1 \%$ triton X-100 and blocked with blocking solution ( $2 \%$ bovine serum albumin, $0.2 \%$ goat serum, and $0.1 \%$ Tween 20 ). Then, the cells were incubated overnight at $4{ }^{\circ} \mathrm{C}$ with primary antibodies (1:50 dilution) against rat CD44 (BD Biosciences, San Jose, CA, USA), CD117 (Merck Millipore, Billerica, MA, USA), and CD34 (Santa Cruz Biotechnology Inc., Dallas, TX, USA). Next day, the cells were washed with PBS three times for 5 minutes each, incubated with secondary antibody conjugated to Alexa Fluor 546 (1:200 dilution) for 1 hour at room temperature, and washed five times with PBS for 5 minutes each. Nuclei were stained with 4',6-diamidino2-phenylindole. Finally, the slides were mounted with a mounting medium and were examined under fluorescent microscope (TE2000 Nikon, Japan).

\section{Flow cytometry}

Flow cytometry analysis was performed to analyze the expression of cell surface markers on BM-MSCs by using flow cytometer (FACS Calibur, Becton Dickinson, USA). BM-MSCs were grown in a tissue culture flask and washed two times with PBS. Cell dissociation buffer (Thermo Fisher Scientific) was used to detach the cells. The cells were then collected and incubated with primary antibodies against $\mathrm{CD} 44$ and $\mathrm{CD} 45$ at $4^{\circ} \mathrm{C}$ for 30 minutes. Unlabeled cells were used as a negative control. Then, the cells were washed three times with flow cytometry solution (bovine serum 
albumin, sodium azide, ethylenediaminetetraacetic acid), stained with secondary antibodies conjugated to Alexa Fluor 546 (1:200), washed, and analyzed using flow cytometer.

\section{Differentiation of BM-MSCs into cardiomyocytes and neuronal-like cells} Treatment of BM-MSCs with 5-azacytidine and zebularine

MSCs were divided into three separate groups: 1) untreated control, 2) MSCs treated with $1 \mu \mathrm{M}$ of 5-azacytidine, and 3) MSCs treated with $1 \mu \mathrm{M}$ of zebularine. The final concentration of each compound in the solution was supplemented to the cells and incubated in $\mathrm{CO}_{2}$ incubator at $37^{\circ} \mathrm{C}$. After overnight incubation, the medium was aspirated, and the cells were washed twice with PBS. New medium was added, and the cells were again incubated for 10 days. The treated cells were monitored under phase contrast microscope (TE2000 Nikon) for morphological changes.

\section{Treatment of BM-MSCs with BME}

MSCs from passage 2 were initially treated for 24 hours with $1 \mathrm{mM}$ final concentration of BME (Thermo Fisher Scientific) in complete DMEM to induce neuronal differentiation. After overnight incubation, the medium was replaced with fresh serum-free medium with $10 \mathrm{mM}$ concentration of BME for 3 hours. The treated cells were monitored under phase contrast microscope to observe the morphological changes.

\section{Immunocytochemistry}

Treated MSCs were processed for immunocytochemistry as described in the case of characterization of MSCs. The treated cells were incubated overnight at $4^{\circ} \mathrm{C}$ with primary antibodies (1:50 dilution) against rat-specific cardiac proteins, GATA 4 (Abcam, Cambridge, MA, USA), Nkx 2.5 (Santa Cruz), and cardiac troponin-T (CTT) (Santa Cruz); and neuronal markers, Flk (Chemicon), Nestin (Abcam), Nef (Abcam), and $\beta$-tubulin (Abcam).

\section{RNA isolation and reverse transcription PCR analysis}

Total RNA was extracted from treated and untreated BM-MSCs using the RNeasy Mini Kit (Qiagen NV, Venlo, the Netherlands) according to the manufacturer's instructions. The purity of the total RNA was determined spectrophotometrically at 260 and $280 \mathrm{~nm}$, with an acceptable $A_{260}: A_{280}$ ratio of 1.8-2.0. The cDNAs were synthesized using Omniscript Reverse Transcription Kit (Qiagen NV) according to the manufacturer's instructions. cDNA was amplified by PCR using the Qiagen PCR kit according to the manufacturer's instructions. PCR cycles were started with initial denaturation for 3 minutes at $93^{\circ} \mathrm{C}$, followed by 35 cycles of denaturation at $93^{\circ} \mathrm{C}(1$ minute), annealing at $62^{\circ} \mathrm{C}$ ( 30 seconds), and extension at $68^{\circ} \mathrm{C}(1$ minute). The PCR products were electrophoretically resolved on $1 \%$ agarose gel. AlphaEase FC software (Alpha Innotech, San Leandro, CA, USA) was used to measure the gel density of the specific bands. Density of each band was normalized relative to glyceraldehyde 3-phosphate dehydrogenase (GAPDH).

\section{Statistical analysis}

Data obtained were presented as mean \pm standard error of the mean. Statistical significance $\left({ }^{*} P<0.05\right)$ was determined by using the SAS 9.1 statistical program (SAS Institute Inc., Cary, NC, USA), and data were subjected to $t$-test to determine significant differences in gene expression level between the differentiated and undifferentiated BM-MSCs.

\section{Results}

\section{Isolation and characterization of BM-MSCs}

MSCs represent nonhematopoietic subpopulation of the bone marrow. These cells displayed a stable phenotype having monolayer in culture. They showed rapid adherence with spindle-shaped morphology and clonal properties till passages 3-4, which decreased with further increase in the passage number. The homogenous population of the cells demonstrated uniform fibroblast-like morphology. Cell surface antigen expression by immunochemistry showed positive expression for CD29, CD44, and CD117, which are known MSC markers, and negative expression for CD45, a hematopoietic cell surface marker (Figure 1). The flow cytometric analysis further revealed that MSCs were positive for CD44 with $>85 \%$, while the hematopoietic marker CD45 was low in expression (Figure 2).

\section{Differentiation of BM-MSCs into cardiomyocytes and neuronal-like cells Morphology of BM-MSCs after 5-azacytadine and zebularine treatment}

After exposure to 5-azacytidine and zebularine, changes in morphology were gradually observed. The treated cells were larger in size. Myotube-like morphology was observed in the cultured cells after 10 days of treatment (Figure 3). These changes suggested the differentiation of BM-MSCs into cardiac-like cells. 

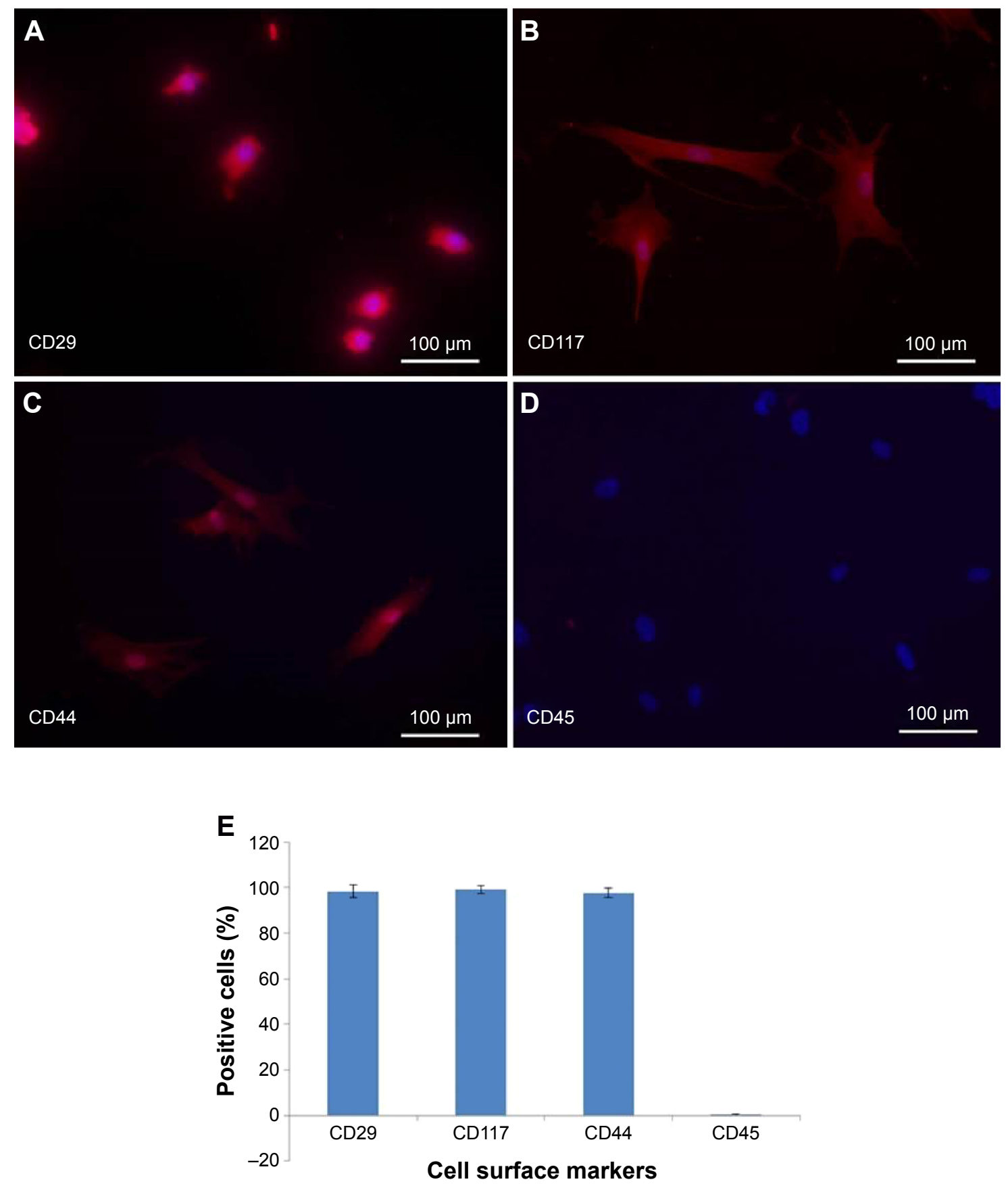

Figure I Immunostaining of bone marrow-derived mesenchymal stem cells (BM-MSCs) on the basis of surface marker expression.

Notes: (A) Expression of CD29, (B) CDII7 (c-Kit), (C) CD44, (D) CD45 as negative control, and (E) quantitative analysis of cell surface markers' expression, indicating that majority of cells are positive for MSC markers and negative for hematopoietic marker. Nuclei were stained with DAPI.

Abbreviation: DAPI, 4',6-diamidino-2-phenylindole.

\section{Morphology of BM-MSCs after BME treatment}

Upon exposure to BME, cells exhibited changes in the morphological features toward typical neurons. BM-MSCs displayed neuronal-like phenotype under phase contrast microscope, having extended processes such as axons and dendrites outgrowing from the cell body, which formed a synaptic connection to the neighboring cells (Figure 4).
These changes suggested the differentiation of BM-MSCs into neuron-like cells.

Expression of cardiac and neuronal-specific genes in the treated cells

The mRNA expression of cardiac- and neuronal-specific markers of the treated and untreated MSCs was assessed by reverse 

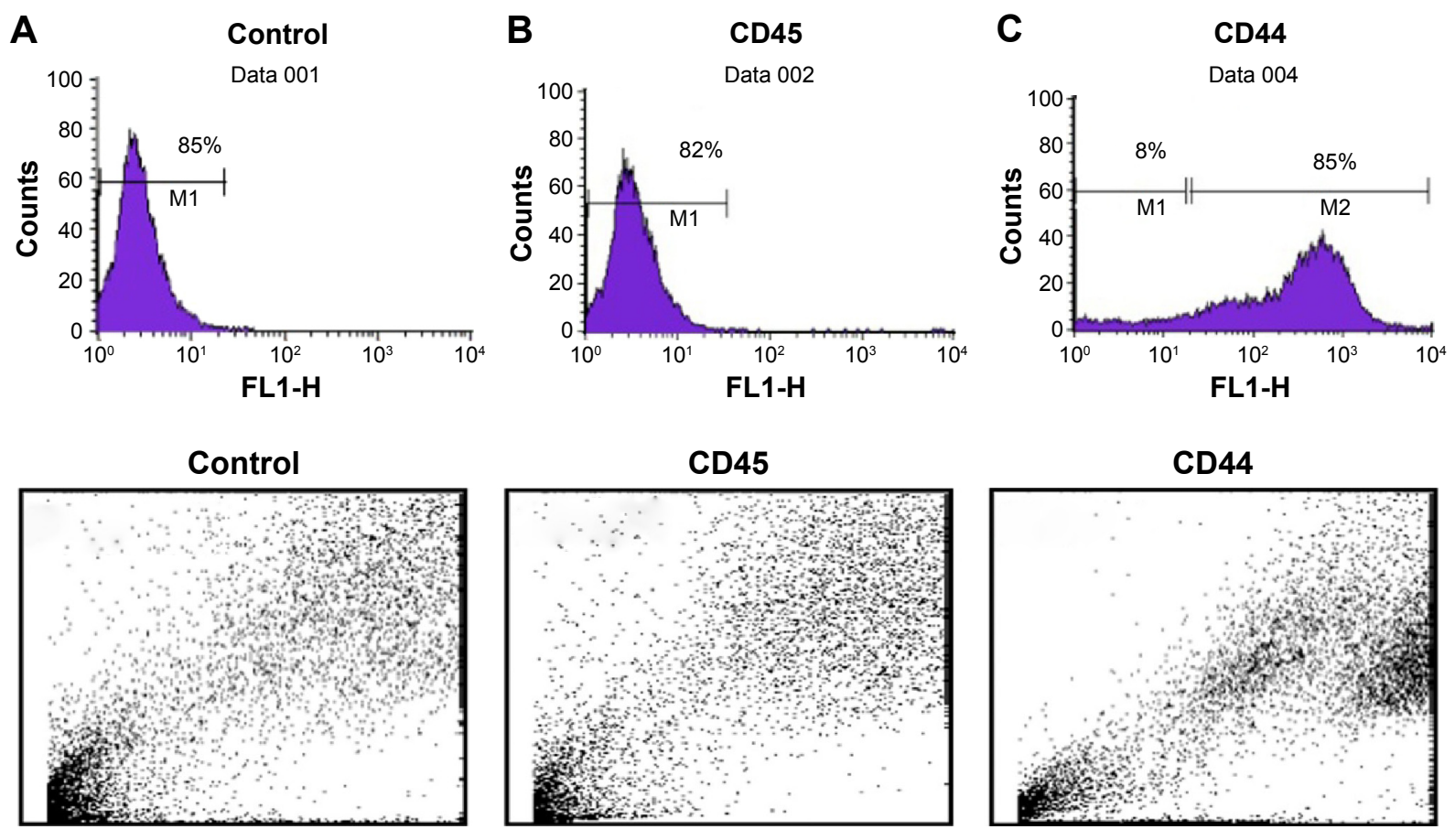

Figure 2 Flow cytometeric analysis of expression of cell surface markers in bone marrow-derived mesenchymal stem cells (BM-MSCs).

Notes: After treatment with specific primary antibodies, cells were analyzed for specific antigens in triplicates. The selected surface markers are shown by the filled histograms as (A) control group or labeled only with Alexa Fluor 546 secondary antibody, (B) MSCs labeled with anti-CD45 antibody (a hematopoietic marker); negative marker, and (C) MSCs labeled with anti-CD44 antibody, positive marker (85\%). The histograms are accompanied by the respective scatter plots.
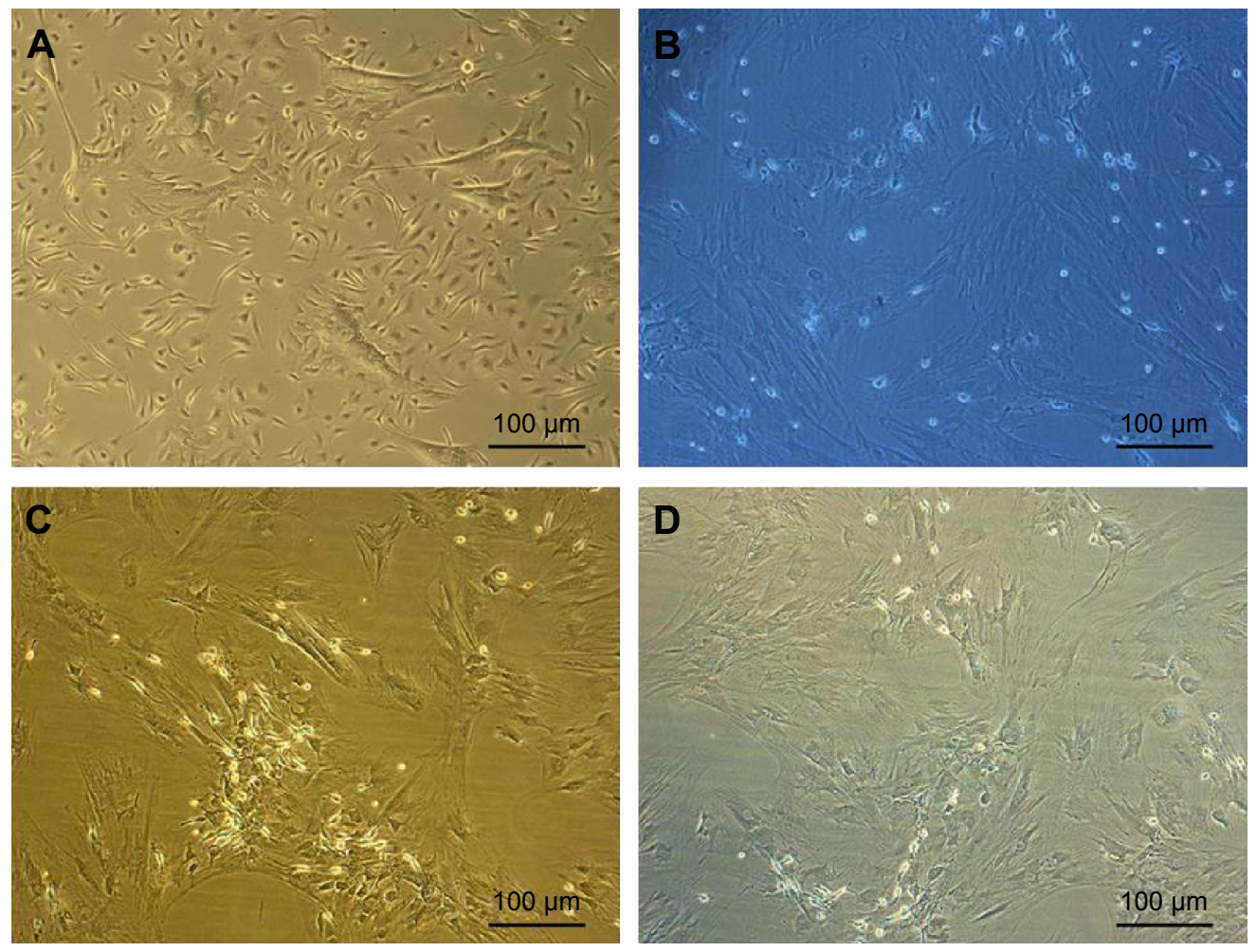

Figure 3 Morphological changes in bone marrow-derived mesenchymal stem cells (BM-MSCs) after treatment with 5-azacytidine and zebularine.

Notes: (A) BM-MSCs show spindle-shaped morphology before treatment at day I, (B) control cells without any treatment for 10 days, (C) MSCs treated with I $\mu$ M 5 -azacytidine for 10 days, and (D) MSCs treated with I $\mu$ M of zebularine for 10 days. The morphology of the MSCs changed after treatment and the cells began to differentiate into cardiomyocytes with increase in cell size and myotube formation. 

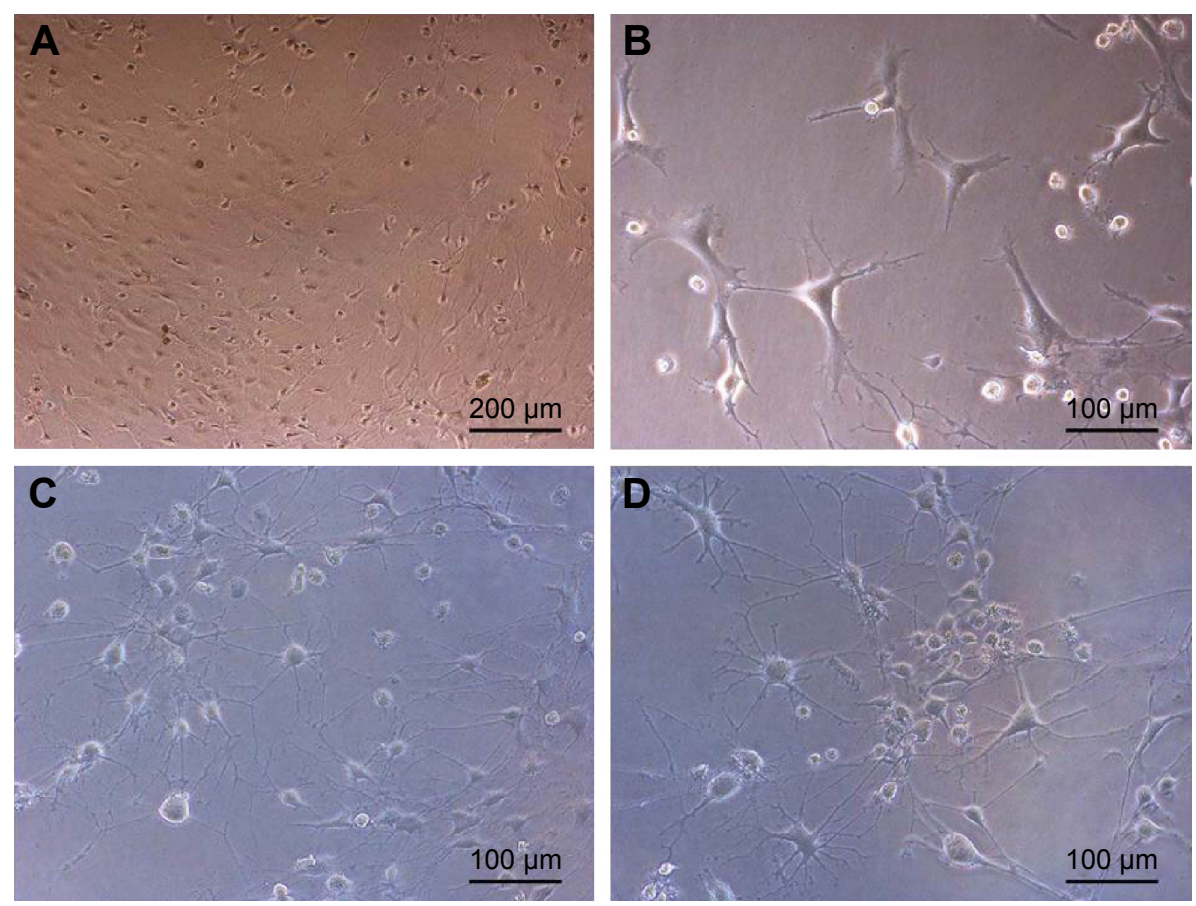

Figure 4 Morphological changes in BM-MSCs after treatment with BME after overnight incubation.

Notes: (A) MSCs with no treatment morphologically appeared spindle shaped; (B) MSCs after treatment with BME started showing changes in cellular morphology, and sprouting of early dendrite-like projection is visible; (C and D) MSCs started forming cell body with visible multipolar dendrite formation and interconnecting network formation with neighboring cells, indicating neuron-like cellular morphology, forming axons and dendrites.

Abbreviations: BM-MSCs, bone marrow-derived mesenchymal stem cells; BME, $\beta$-mercaptoethanol.

transcriptase-polymerase chain reaction (RT-PCR). The housekeeping gene, GAPDH, was used for internal control. The amplicon of GAPDH was found in both treated and untreated MSCs, indicating that GAPDH remained activated. RT-PCR analysis revealed the presence of cardiac alpha myosin heavy chain (MHC), cardiac transcription factor (GATA-4), and Nkx2.5 in treated MSCs (Figure 5). Additionally, very low intensity bands of selected cardiac-specific genes were also observed in untreated MSCs except in the case of GATA-4. Relative quantification of each band was performed using gel documentation system; integrated density value of each band was quantified, normalized with GAPDH, and compared with the control group. The gene expression levels of $\mathrm{MHC}, \mathrm{Nkx} 2.5$, and GATA-4 were significantly higher after treatment compared with control (Figure 5). RT-PCR analysis also revealed the presence of neuronal-specific markers in treated MSCs. Both control and treated cells expressed Nestin, microtubuleassociated protein (Map2), Tau, and Nef. However, the expression levels of these markers were significantly higher in treated cells compared with control MSCs as shown in Figure 6.

\section{Expression of cardiac and neuronal-specific proteins in the treated cells}

After separate treatments with 5-azacytidine and zebularine at $1 \mu \mathrm{M}$ concentrations, MSCs continued to proliferate and differentiate. After 10 days in culture, the cells showed extended cytoplasmic processes (Figure 3C and D). Immunocytochemical analysis of 5-azacytadine and zebularinetreated MSCs showed expression of GATA4, Nkx 2.5, and CTT as shown in Figure 7. Immunostaining of cells treated with 5-azacytadine and zebularine revealed that majority of the cells express cardiac proteins, indicating that the stromal cells are differentiated into cardiomyocytes.

MSCs after induction to neurons in neuronal differentiation medium after overnight incubation were immunostained for assessing the expression of neuronal markers, Flk, Nef, Nestin, and $\beta$-tubulin as shown in Figure 8.

\section{Discussion}

It is challenging to anticipate the differentiation of uncommitted stem cells, which could result in other undesirable cell types after delivery to the targeted tissue. ${ }^{35}$ Studies have shown that pretreatment of stem cells could be a better alternative strategy for overcoming uncontrolled differentiation. Several studies have shown that chemical compounds such as ascorbic acid, retinoic acid, BME, and 5-azacytidine have the potential to differentiate stem cells into other types of cells, including cardiac and neuronal-like cells, although results from different studies are contradictory and hence inconclusive. .,28,36-43 $^{-4}$ 
A

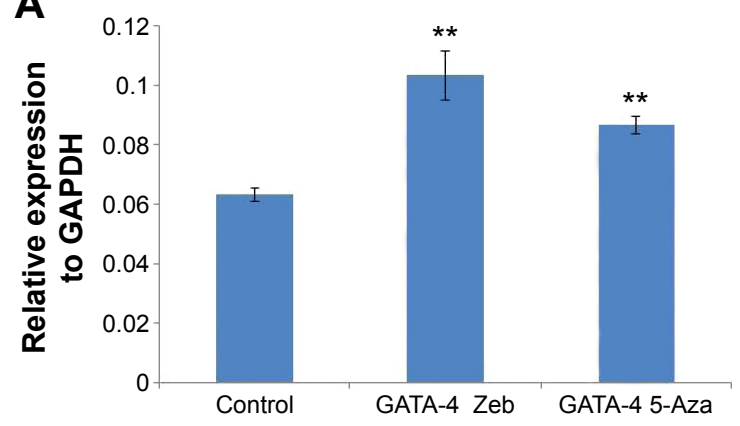

B

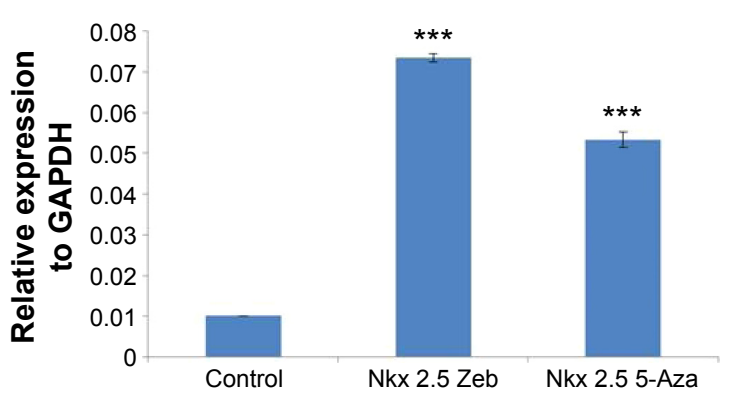

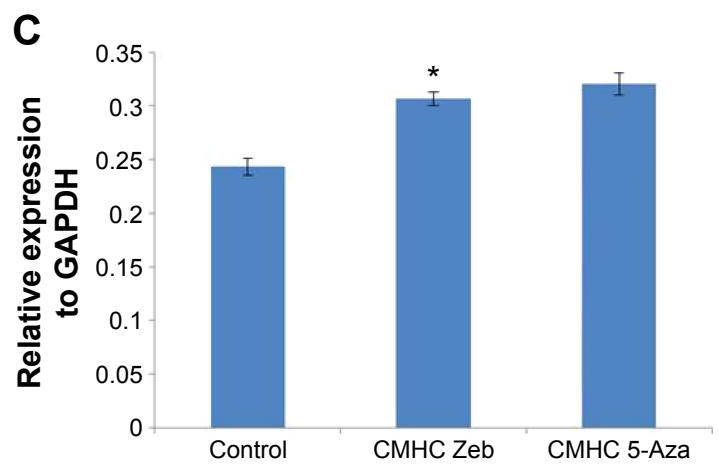

Figure 5 RT-PCR analysis of cardiac-specific gene expression in treated and untreated BM-MSCs.

Notes: Expression of (A) GATA-4, (B) CTT, and (C) Nkx2.5 was observed and normalized with GAPDH that expressed in both untreated and treated MSCs. Gene expression levels of cardiac-specific genes in 5-azacytidine- and zebularine-treated cells were compared to relative control group ( $n=3$, level of significance $P<0.05$; $* P<0.05$; $* * P<0.01$; and $* * * P<0.001$ ).

Abbreviations: RT-PCR, reverse transcriptase polymerase chain reaction; BM-MSCs, bone marrow-derived mesenchymal stem cells; GAPDH, glyceraldehyde 3-phosphate dehydrogenase; CTT, cardiac troponin-T.

\section{A Tau expression in MSCs after treatment}

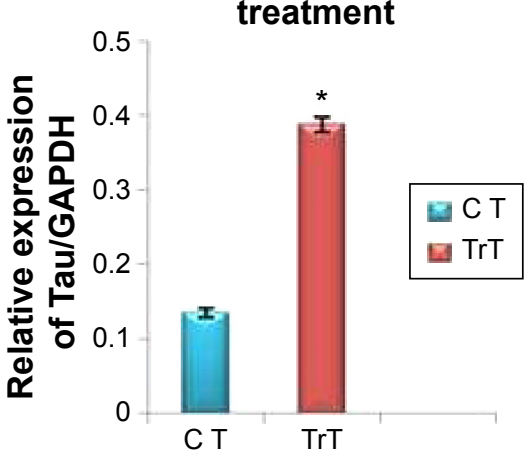

\section{Map2 expression in MSCs after treatment}

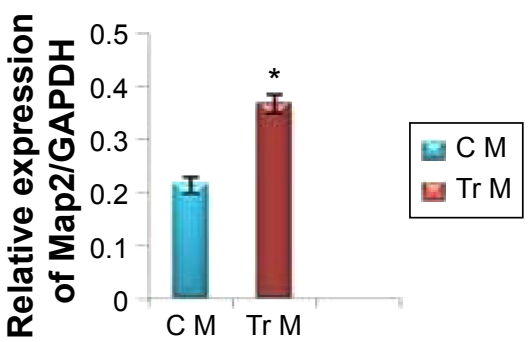

\section{B Nestin expression in MSCs} after treatment

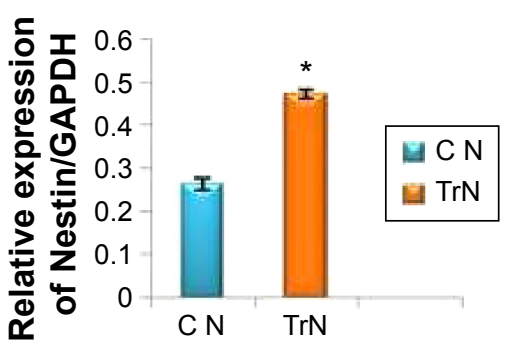

\section{Nefl expression in MSCs after treatment}

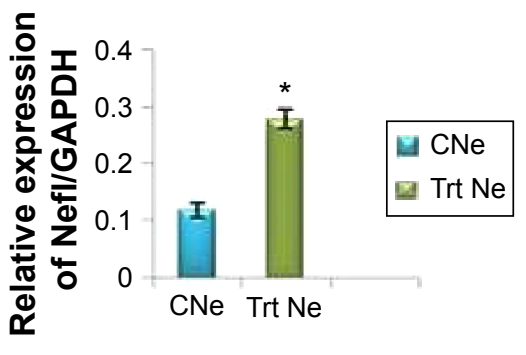

Figure 6 Gene expression levels of BM-MSCs differentiated to neurons after $\beta$-mercaptoethanol (BME) treatment.

Notes: Expression of (A) Tau, (B) Nestin, (C) Map2, and (D) Nefl was detected in treated cells. Densitometric analysis of gene expression levels of neuronal-specific genes was normalized with GAPDH and compared with relative control group $(n=3$, level of significance $P<0.05 ; * P<0.05 ; * * P<0.01$; and $* * * P<0.00 \mathrm{I})$. Abbreviations: BM-MSCs, bone marrow-derived mesenchymal stem cells; GAPDH, glyceraldehyde 3-phosphate dehydrogenase. 

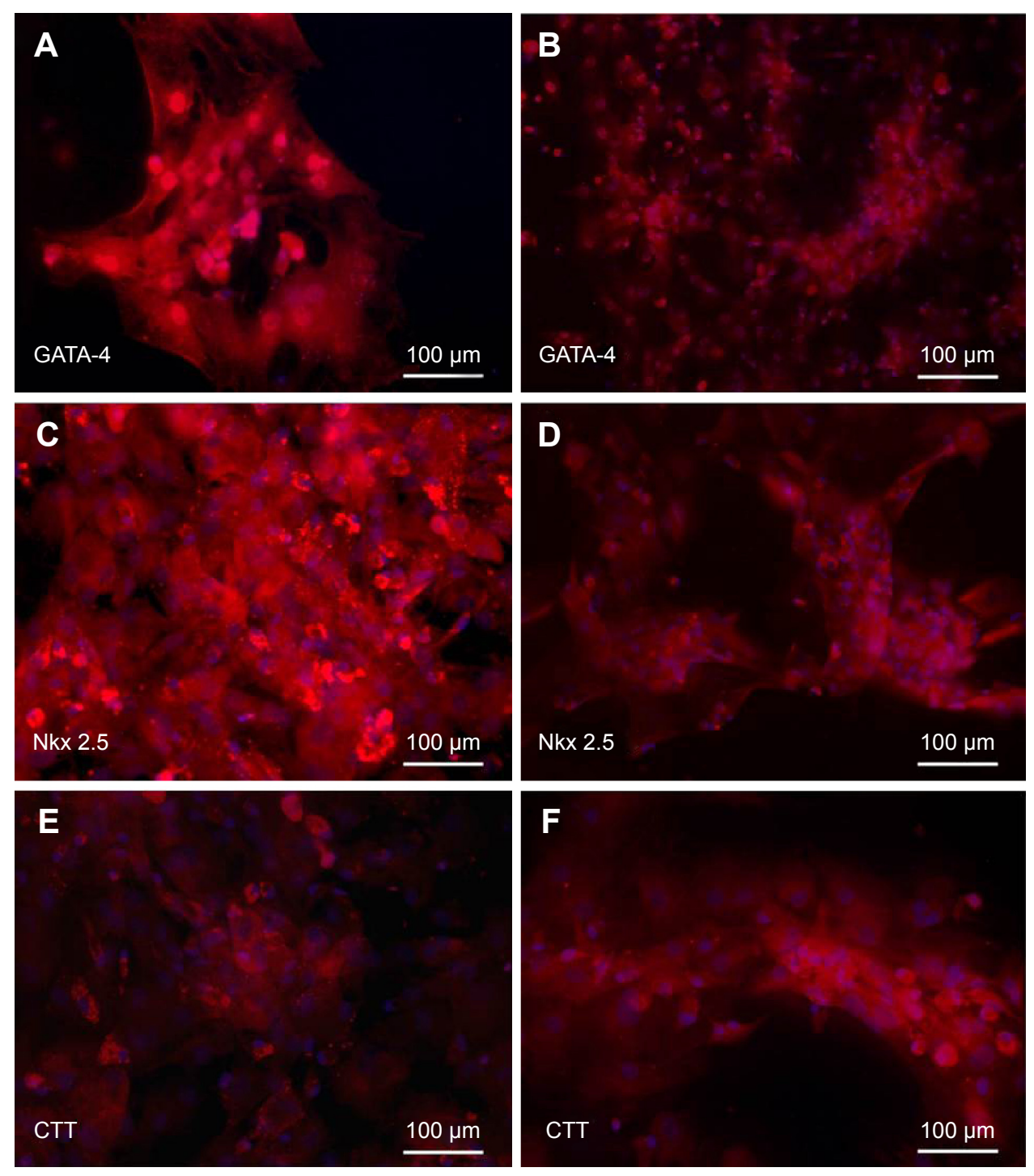

Figure 7 Immunostaining of BM-MSCs differentiated to cardiac-like cells after treatment with I $\mu$ M of 5-azacytadine and zebularine.

Notes: (A and B) GATA-4, (C and D) Nkx 2.5, and (E and F) cardiac troponin-T (CTT) expression after 5-azacytadine and zebularine treatment, respectively. Nuclei were stained with DAPI.

Abbreviations: BM-MSCs, bone marrow-derived mesenchymal stem cells; DAPI, 4',6-diamidino-2-phenylindole.

In this study, after successful isolation and characterization of BM-MSCs on the basis of cell surface markers and also on the basis of osteogenic and adipogenic differentiation as reported earlier, ${ }^{44}$ cultured BM-MSCs were treated separately with 5-azacytidine, zebularine, and BME. Prior to treatment, concentrations of 5-azacytidine and zebularine were optimized and selected on the basis of having less number of apoptotic cells as reported earlier. ${ }^{28}$ Treatment with 5-azacytidine and zebularine was expected to induce differentiation of BM-MSCs into cardiomyocytes while that with BME would result in the differentiation into neuronal-like cells. It is hypothesized that the treatment of cells by 5 -azacytidine makes the cells less responsive to other inductive factors secreted by the microenvironment that might modulate the differentiation. ${ }^{36}$ However, the toxicity and instability of 5-azacytidine are high, which could be unsafe for the biological system. Zebularine, a cytidine analog containing a 2-pyrimidinone ring, is another novel DNA methyltransferase inhibitor, which has been developed as a more stable and less toxic drug compared to 5 -azacytidine..$^{28,34}$ There is a less evidence of evaluating the differentiation potential of zebularine on BM-MSCs compared to well-known compounds such as 5-azacytidine. ${ }^{42,43,45}$ Zebularine was studied in microbial systems for cancer therapy using mammalian cell lines. ${ }^{46} \mathrm{We}$ earlier reported the differentiation potential of zebularine in an in vitro study. The present study shows improvement in the differentiation strategy using even lesser concentration of the compound, 

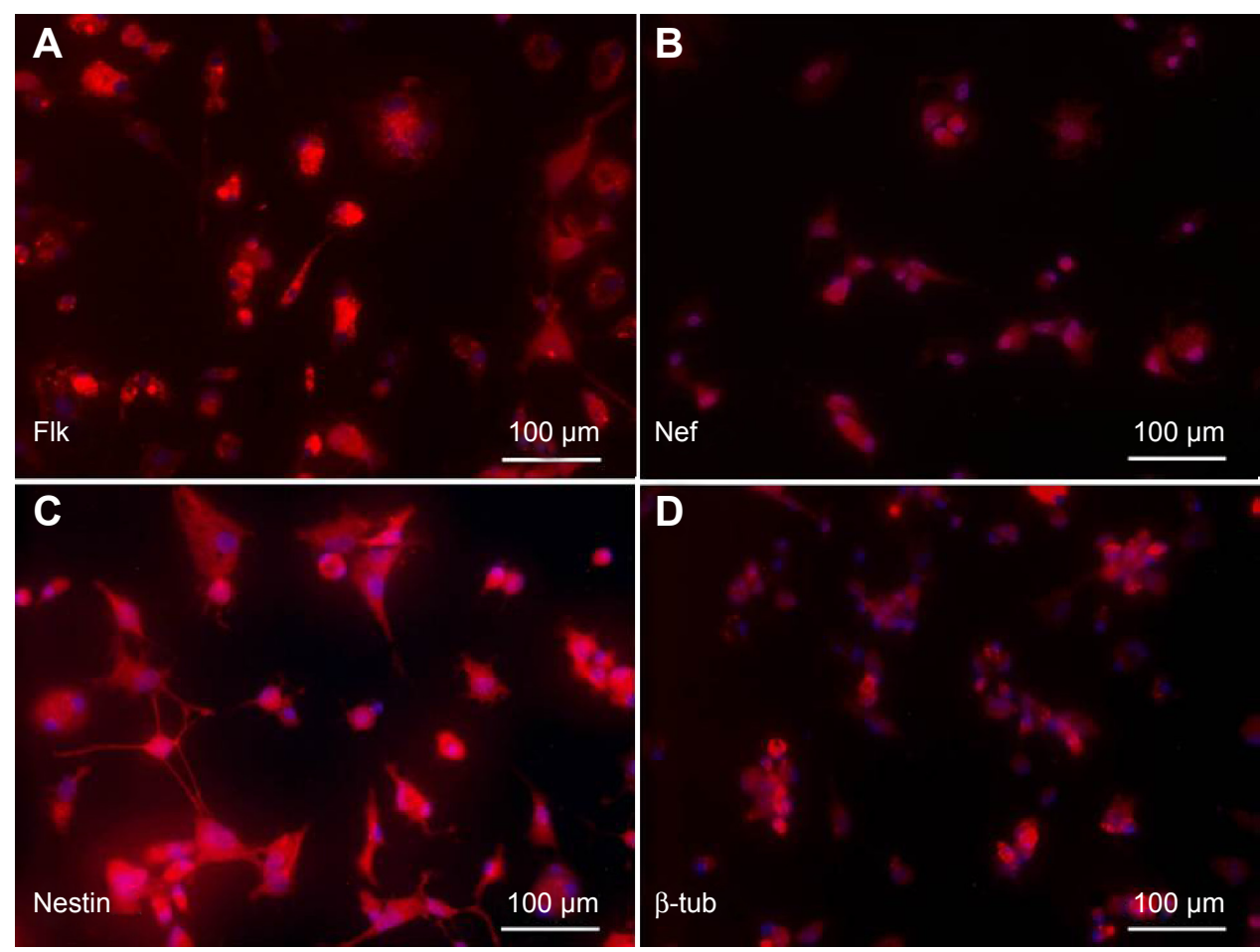

Figure 8 Immunostaining of BM-MSCs differentiated to neurons after $\beta$-mercaptoethanol (BME) treatment.

Notes: Treated cells were positive for neuronal markers (A) Flk, (B) Nef, (C) Nestin, and (D) $\beta$-tubulin. Nuclei were stained with DAPI. Abbreviations: BM-MSCs, bone marrow-derived mesenchymal stem cells; DAPI, 4',6-diamidino-2-phenylindole.

so the chances of toxicity can be minimized. Furthermore, we also observed that differentiation to mature cell type needs lesser time period; thus, differentiated cells can be readily available for transplantation. However, further studies are needed to assess the molecular mechanism of zebularine into cardiomyocytes after transplantation in in vivo models. The demethylating agent, zebularine, promotes changes in BM-MSCs, which in turn causes differentiation of treated MSC cells into cardiac-like cells. Morphologically, fibroblast-like cells gradually increased in size showing myotube-like structure after exposure to zebularine. Notably, data from RT-PCR and immunocytochemistry revealed the presence of cardiacspecific genes and proteins, respectively, including $\mathrm{Nkx}$ 2.5, MHC, and cardiac transcription factor (GATA-4), after treatment with 5-azacytidine and zebularine. GATA-4 and $\mathrm{Nkx} 2.5$ are known as key regulators in cardiac development, ${ }^{46}$ and GATA-4 is expressed during myocardium development. ${ }^{47}$ GATA transcription factors are involved in the expression of several genes, which encode contractile proteins such as CTT and cardiac alpha actin. ${ }^{48}$ It can be hypothesized that pretreatment of BM-MSCs with zebularine can also facilitate the differentiation toward cardiomyogenic lineage under in vivo conditions. Therefore, zebularine could be a good candidate to be used as an alternative to 5-azacytidine, which is less stable and more toxic.

BME is another well-known antioxidant, which is being used as inducer to differentiate MSCs into neuronal-like cells. Different studies have used BME with different protocols and medium condition. ${ }^{4,7}$ In the study by Woodbury et al, ${ }^{4}$ BM-

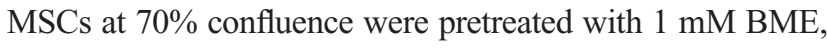
which contained basic fibroblast growth factor for 24 hours. The cells were then cultured in DMEM, which was supplemented with dimethyl sulfoxide, butylated hydroxyanisole, valproic acid, forskolin, insulin, hydrocortisone, and $\mathrm{KCl}$, for 6 days. ${ }^{7}$

To the best of our knowledge, most of the studies have used a mixture of BME and certain growth factors and cytokines in order to differentiate MSCs into neuronal cells. We treated cultured MSCs with BME in two different medium conditions; MSCs were first treated with serum-enriched DMEM with $1 \mathrm{mM}$ of BME for 24 hours and consequently replaced with serum-free DMEM media with $10 \mathrm{mM}$ of BME for 3 hours. As mentioned earlier, several studies have described different methods of chemical induction for neuronal differentiation; however, results from different studies are contradictory and hence inconclusive and mostly showed less number of interconnecting neurons. ${ }^{29,38}$ In our study, MSCs were morphologically differentiated into neuronal-like cells after second induction. Untreated MSCs exhibited fibroblast-like cells in 
the control group. Neuronal-like cells were distinctively distinguished by highly refractive cell bodies (soma) with neuronlike processes that resembled axons and dendrites. Most of these dendrites showed interconnections with neighboring cells. Differentiated neuronal-like cells were further confirmed by the presence of neuronal-specific markers, including Map2, Nestin, Nefl, and Tau by RT-PCR, and immunostaining at the gene and protein levels, respectively. Nestin is classically considered as a specific marker of neuronal stem cells, and NSE and Map2 are other neuronal markers. Our results are parallel with previously conducted studies that showed the neuronal differentiation potential of MSCs isolated from different tissue origins. ${ }^{7,49,50}$ A majority of laboratories have used combination of cytokines and growth factors for neuronal induction of MSCs. ${ }^{4,7,51,52}$ Whereas in this study, no growth factors or cytokines were used. Our results indicate that it is possible to derive interconnecting neuron-like cells from BM-MSCs with identical morphology and the expression of neuronal-specific markers.

\section{Acknowledgments}

The authors would like to thank the University of Malaya for providing the research grant, High Impact Research (J-21010-73877) and FP040-2014A.

\section{Disclosure}

The authors report no conflicts of interest in this work.

\section{References}

1. Elnakish MT, Hassan F, Dakhlallah D, Marsh CB, Alhaider IA, Khan M. Mesenchymal stem cells for cardiac regeneration: translation to bedside reality. Stem Cells Int. 2012;2012:14

2. Lu J, Moochhala S, Moore XL, et al. Adult bone marrow cells differentiate into neural phenotypes and improve functional recovery in rats following traumatic brain injury. Neurosci Lett. 2006;398(1):12-17.

3. Melo LG, Pachori AS, Kong D, et al. Molecular and cell-based therapies for protection, rescue, and repair of ischemic myocardium reasons for cautious optimism. Circulation. 2004;109(20):2386-2393.

4. Woodbury D, Schwarz EJ, Prockop DJ, Black IB. Adult rat and human bone marrow stromal cells differentiate into neurons. J Neurosci Res. 2000;61(4):364-370.

5. Mendis S, Puska P, Norrving B. Global Atlas on Cardiovascular Disease Prevention and Control. Geneva, Switzerland: World Health Organization; 2011.

6. Laflamme MA, Murry CE. Regenerating the heart. Nat Biotechnol. 2005;23(7):845-856.

7. Kumar BM, Maeng GH, Lee YM, et al. Neurogenic and cardiomyogenic differentiation of mesenchymal stem cells isolated from minipig bone marrow. Res Vet Sci. 2012;93(2):749-757.

8. Iso Y, Spees JL, Serrano C, et al. Multipotent human stromal cells improve cardiac function after myocardial infarction in mice without long-term engraftment. Biochem Biophys Res Commun. 2007;354(3): 700-706.

9. Cho J, Zhai P, Maejima Y, Sadoshima J. Myocardial injection with GSK-3 $\beta$-overexpressing bone marrow-derived mesenchymal stem cells attenuates cardiac dysfunction after myocardial infarction. Circ Res. 2011; 108(4):478-489.
10. Ortiz LA, Dutreil M, Fattman C, et al. Interleukin 1 receptor antagonist mediates the antiinflammatory and antifibrotic effect of mesenchymal stem cells during lung injury. Proc Natl Acad Sci U S A. 2007;104(26): 11002-11007.

11. Curley GF, Hayes M, Ansari B, et al. Mesenchymal stem cells enhance recovery and repair following ventilator-induced lung injury in the rat. Thorax. 2011;67(6):496-501.

12. Kunter U, Rong S, Djuric Z, et al. Transplanted mesenchymal stem cells accelerate glomerular healing in experimental glomerulonephritis. J Am Soc Nephrol. 2006;17(8):2202-2212.

13. Alfarano C, Roubeix C, Chaaya R, et al. Intraparenchymal injection of bone marrow mesenchymal stem cells reduces kidney fibrosis after ischemia-reperfusion in cyclosporine-immunosuppressed rats. Cell Transplant. 2012;21(9):2009-2019.

14. Lee RH, Seo MJ, Reger RL, et al. Multipotent stromal cells from human marrow home to and promote repair of pancreatic islets and renal glomeruli in diabetic NOD/scid mice. Proc Natl Acad Sci U S A. 2006; 103(46):17438-17443.

15. Si Y, Zhao Y, Hao H, et al. Infusion of mesenchymal stem cells ameliorates hyperglycemia in Type 2 diabetic rats identification of a novel role in improving insulin sensitivity. Diabetes. 2012;61(6):1616-1625.

16. Kanazawa H, Fujimoto Y, Teratani T, et al. Bone marrow-derived mesenchymal stem cells ameliorate hepatic ischemia reperfusion injury in a rat model. PLoS One. 2011;6(4):e19195.

17. Zhao W, Li JJ, Cao DY, et al. Intravenous injection of mesenchymal stem cells is effective in treating liver fibrosis. World J Gastroenterol. 2012;18(10):1048.

18. Forostyak S, Jendelova P, Sykova E. The role of mesenchymal stromal cells in spinal cord injury, regenerative medicine and possible clinical applications. Biochimie. 2013;95(12):2257-2270.

19. Schaakxs D, Kalbermatten DF, Raffoul W, Wiberg M, Kingham PJ. Regenerative cell injection in denervated muscle reduces atrophy and enhances recovery following nerve repair. Muscle Nerve. 2013;47(5): 691-701.

20. Orlic D, Kajstura J, Chimenti S, Bodine DM, Leri A, Anversa P. Bone marrow stem cells regenerate infarcted myocardium. Pediatr Transplant. 2003;7(s3):86-88.

21. Lindvall O, Kokaia Z, Martinez-Serrano A. Stem cell therapy for human neurodegenerative disorders - how to make it work. Nat Med. 2004; 10:S42-S50.

22. Antonitsis P, Ioannidou-Papagiannaki E, Kaidoglou A, Papakonstantinou C. In vitro cardiomyogenic differentiation of adult human bone marrow mesenchymal stem cells. The role of 5-azacytidine. Interact Cardiovasc Thorac Surg. 2007;6(5):593-597.

23. García-Castro J, Trigueros C, Madrenas J, Perez-Simon J, Rodriguez R, Menendez P. Mesenchymal stem cells and their use as cell replacement therapy and disease modelling tool. J Cell Mol Med. 2008;12(6b): 2552-2565.

24. Psaltis PJ, Zannettino AC, Worthley SG, Gronthos S. Concise review: mesenchymal stromal cells: potential for cardiovascular repair. Stem Cells. 2008;26(9):2201-2210.

25. Qian Q, Qian H, Zhang X, et al. 5-Azacytidine induces cardiac differentiation of human umbilical cord-derived mesenchymal stem cells by activating extracellular regulated kinase. Stem Cells Dev. 2011;21(1): 67-75.

26. Zainal Ariffin SH, Kermani S, Zainol Abidin IZ, et al. Differentiation of dental pulp stem cells into neuron-like cells in serum-free medium. Stem Cells Int. 2013;2013:10.

27. Zeng R, Wang L-W, Hu Z-B, et al. Differentiation of human bone marrow mesenchymal stem cells into neuron-like cells in vitro. Spine. 2011;36(13):997-1005.

28. Naeem N, Haneef K, Kabir N, Iqbal H, Jamall S, Salim A. DNA methylation inhibitors, 5-azacytidine and zebularine potentiate the transdifferentiation of rat bone marrow mesenchymal stem cells into cardiomyocytes. Cardiovasc Ther. 2013;31(4):201-209.

29. Cruz Villagrán C, Amelse L, Neilsen N, Dunlap J, Dhar M. Differentiation of equine mesenchymal stromal cells into cells of neural lineage: potential for clinical applications. Stem Cells Int. 2014;2014:12. 
30. Toma C, Pittenger MF, Cahill KS, Byrne BJ, Kessler PD. Human mesenchymal stem cells differentiate to a cardiomyocyte phenotype in the adult murine heart. Circulation. 2002;105(1):93-98.

31. Xu W, Zhang X, Qian H, et al. Mesenchymal stem cells from adult human bone marrow differentiate into a cardiomyocyte phenotype in vitro. Exp Biol Med. 2004;229(7):623-631.

32. Dimarakis I, Levicar N, Nihoyannopoulos P, Gordon MY, Habib NA. In vitro stem cell differentiation into cardiomyocytes: part 2: chemicals, extracellular matrix, physical stimuli and coculture assays. JCardiothorac Renal Res. 2006;1(2):115-121.

33. Ye N-S, Zhang R-L, Zhao Y-F, Feng X, Wang Y-M, Luo G-A. Effect of 5-azacytidine on the protein expression of porcine bone marrow mesenchymal stem cells in vitro. Genomics Proteomics Bioinformatics 2006;4(1):18-25.

34. Yoo C, Cheng J, Jones P. Zebularine: a new drug for epigenetic therapy. Biochem Soc Trans. 2004;32(6):910-912.

35. Balsam LB, Wagers AJ, Christensen JL, Kofidis T, Weissman IL, Robbins RC. Haematopoietic stem cells adopt mature haematopoietic fates in ischaemic myocardium. Nature. 2004;428(6983):668-673.

36. Rosca A-M, Burlacu A. Effect of 5-azacytidine: evidence for alteration of the multipotent ability of mesenchymal stem cells. Stem Cells Dev. 2010;20(7):1213-1221.

37. Ding S, Schultz PG. A role for chemistry in stem cell biology. Nat Biol. 2004;22(7):833-840.

38. Sanchez-Ramos J, Song S, Cardozo-Pelaez F, et al. Adult bone marrow stromal cells differentiate into neural cells in vitro. Exp Neurol. 2000; 164(2):247-256.

39. Rastegar F, Shenaq D, Huang J, et al. Mesenchymal stem cells: molecular characteristics and clinical applications. World J Stem Cells. 2010;2(4):67.

40. Williams AR, Trachtenberg B, Velazquez DL, et al. Intramyocardial stem cell injection in patients with ischemic cardiomyopathy functional recovery and reverse remodeling. Circ Res. 2011;108(7):792-796.

41. Pan Y, Cai S. Current state of the development of mesenchymal stem cells into clinically applicable Schwann cell transplants. Mol Cell Biochem. 2012;368(1-2):127-135.
42. Burlacu A, Rosca AM, Maniu H, et al. Promoting effect of 5-azacytidine on the myogenic differentiation of bone marrow stromal cells. Eur $J$ Cell Biol. 2008;87(3):173-184.

43. Liu J, Hong L, Zhong X, Chen J, Chen Y. Effects of serum from myocardial infarction rats on rat bone marrow mesenchymal stem cell differentiation into cardiomyocytes. J Clin Rehab Tissue Eng Res. 2009; 13(14):2797-2800.

44. Haneef K, Lila N, Benadda S, Legrand F, Carpentier A, Chachques JC. Development of bioartificial myocardium by electrostimulation of 3D collagen scaffolds seeded with stem cells. Heart Int. 2017;7:e14.

45. Liu Y, Song J, Liu W, Wan Y, Chen X, Hu C. Growth and differentiation of rat bone marrow stromal cells: does 5-azacytidine trigger their cardiomyogenic differentiation? Cardiovasc Res. 2003;58(2):460-468.

46. Cheng JC, Matsen CB, Gonzales FA, et al. Inhibition of DNA methylation and reactivation of silenced genes by zebularine. J Natl Cancer Inst. 2003;95(5):399-409.

47. Planat-Bénard V, Menard C, André M, et al. Spontaneous cardiomyocyte differentiation from adipose tissue stroma cells. Circ Res. 2004;94(2): 223-229.

48. Shirinsky V, Khapchaev AY, Stepanova O. Molecular mechanisms of cardiomyogenesis and the prospects for cardiomyocyte regeneration in cardiac failure. Mol Biol. 2008;42(5):762-772.

49. Rismanchi N, Floyd CL, Berman RF, Lyeth BG. Cell death and long-term maintenance of neuron-like state after differentiation of rat bone marrow stromal cells: a comparison of protocols. Brain Res. 2003;991(1):46-55.

50. Park BW, Kang DH, Kang EJ, et al. Peripheral nerve regeneration using autologous porcine skin-derived mesenchymal stem cells. J Tissue Eng Regen Med. 2012;6(2):113-124.

51. Hermann A, Liebau S, Gastl R, et al. Comparative analysis of neuroectodermal differentiation capacity of human bone marrow stromal cells using various conversion protocols. J Neurosci Res. 2006;83(8): 1502-1514.

52. Zwart I, Hill AJ, Girdlestone J, et al. Analysis of neural potential of human umbilical cord blood-derived multipotent mesenchymal stem cells in response to a range of neurogenic stimuli. J Neurosci Res. 2008;86(9): 1902-1915.

\section{Publish your work in this journal}

Drug Design, Development and Therapy is an international, peerreviewed open-access journal that spans the spectrum of drug design and development through to clinical applications. Clinical outcomes, patient safety, and programs for the development and effective, safe, and sustained use of medicines are a feature of the journal, which has also been accepted for indexing on PubMed Central. The manuscript management system is completely online and includes a very quick and fair peer-review system, which is all easy to use. Visit http://www.dovepress.com/testimonials.php to read real quotes from published authors. 